\title{
Optimization of Experimental Parameters for Electron Capture Dissociation of Peptides in a Fourier Transform Mass Spectrometer
}

\author{
T.-W. Dominic Chan and W. H. Herman Ip \\ Department of Chemistry, The Chinese University of Hong Kong, Shatin, Hong Kong
}

\begin{abstract}
This paper describes our effort in optimizing the experimental parameters for electron capture dissociation (ECD) of peptides in a commercially available Fourier-transform mass spectrometer. Using a built-in electrically heated filament electron gun, it was demonstrated that good quality ECD spectra of peptides (MW < 2500) could be obtained by irradiating the isolated peptide molecule-ions with a short pulse $(50 \mathrm{~ms})$ of low-energy $(3-6 \mathrm{eV})$ electrons. In addition, we have also demonstrated that pulsing of inert cooling gas (argon) could further improve the intensity of the ECD-induced fragment ions. Due presumably to the influence of the strong magnetic field on the trajectories of electrons, the distance between the electron gun and the trapped-ion cell (i.e., $108 \mathrm{~mm}$ versus $20 \mathrm{~mm}$ ) was found to have little influence on the efficiency of the ECD process(es). From a systematic study on the impact of the filament heating current, filament bias voltage, and electron irradiation time on the intensities of precursor ions and various fragment ions, it was postulated that subsequent capture of electrons by the fragment ions, i.e., neutralization of the fragment ions, might be a significant event for limiting the intensity of the fragment ions. (J Am Soc Mass Spectrom 2002, 13, 1396-1406) (C) 2002 American Society for Mass Spectrometry
\end{abstract}

$\mathrm{T}$ landem mass spectrometry is an important technique for generating primary sequences for biomolecules such as peptides/proteins and DNA fragments. In a tandem mass spectrometry experiment, an ion of interest (the so-called "precursor ion") is first isolated from other ions of different $\mathrm{m} / \mathrm{z}$ and is then energized internally to cause unimolecular dissociation. Common methods for ion activation include low (eV)and high (keV)-energy collision-induced dissociation (CID) [1], infrared multiphoton dissociation (IRMPD) [2], blackbody infrared radiative dissociation (BIRD) [3], surface-induced dissociation (SID) [4], and ultraviolet photodissociation (UV-PD) [5]. For peptide analysis, all these methods result mainly in cleavage of the peptide amide bonds, leading to the N-terminal b-type and C-terminal y-type ions [6]. Since the implanted energy is normally randomized in various vibrational degrees of freedoms prior to fragmentation, bond cleavage tends to occur primarily at the weakest linkages in the peptides, such as on the N-terminal side of a proline residue or on the C-terminal side of aspartic acid [7]. As a consequence, complete peptide sequence is rarely obtained from a tandem mass spectrum using the above ion activation method.

Published online October 24, 2002

Address reprint requests to Dr. T.-W. D. Chan, Department of Chemistry, The Chinese University of Hong Kong, Shatin, N.T., Hong Kong. E-mail: twdchan@cuhk.edu.hk
Electron Capture Dissociation (ECD) was first introduced [8] by McLafferty and co-workers in 1998 and is a relatively new mass spectrometry technique for inducing dissociation of multiply-charged ions. By irradiating the multiply-charged peptide/protein ions with low-energy $(\leq 0.2 \mathrm{eV})$ electrons, the peptide/protein ions would undergo exothermic capture of electrons and thus leading to the formation of a reduced radical ion, $[\mathrm{M}+\mathrm{nH}]^{(\mathrm{n}-1) \cdot+}$, which rapidly dissociates via backbone $\mathrm{N}-\mathrm{C}$ cleavage to form predominantly series of c-type and z-type ions. As a result of the non-ergodic nature of ECD process [8], strong backbone bonds can also be cleaved even in the presence of much weaker bonds [9]. It has been demonstrated that this dissociation technique offers more extensive and nonspecific fragmentation, resulting in more extensive sequence information. For instance, collision-induced dissociation of multiply-charged ubiquitin, $8.6 \mathrm{kDa}$, resulted in only $25 \%$ of sequence specific information, whereas electron capture dissociation on collisional-activated ubiquitin ions could lead to complete sequence information [10].

Although it has previously been shown that this dissociation technique can provide important structural information for characterization of labile post-translational modifications $[9,11-15]$ and for de novo sequencing [16], it has not been widely practiced and is limited mainly to a few laboratories with home-made FTMS systems. The lack of positive feedback on this dissocia- 


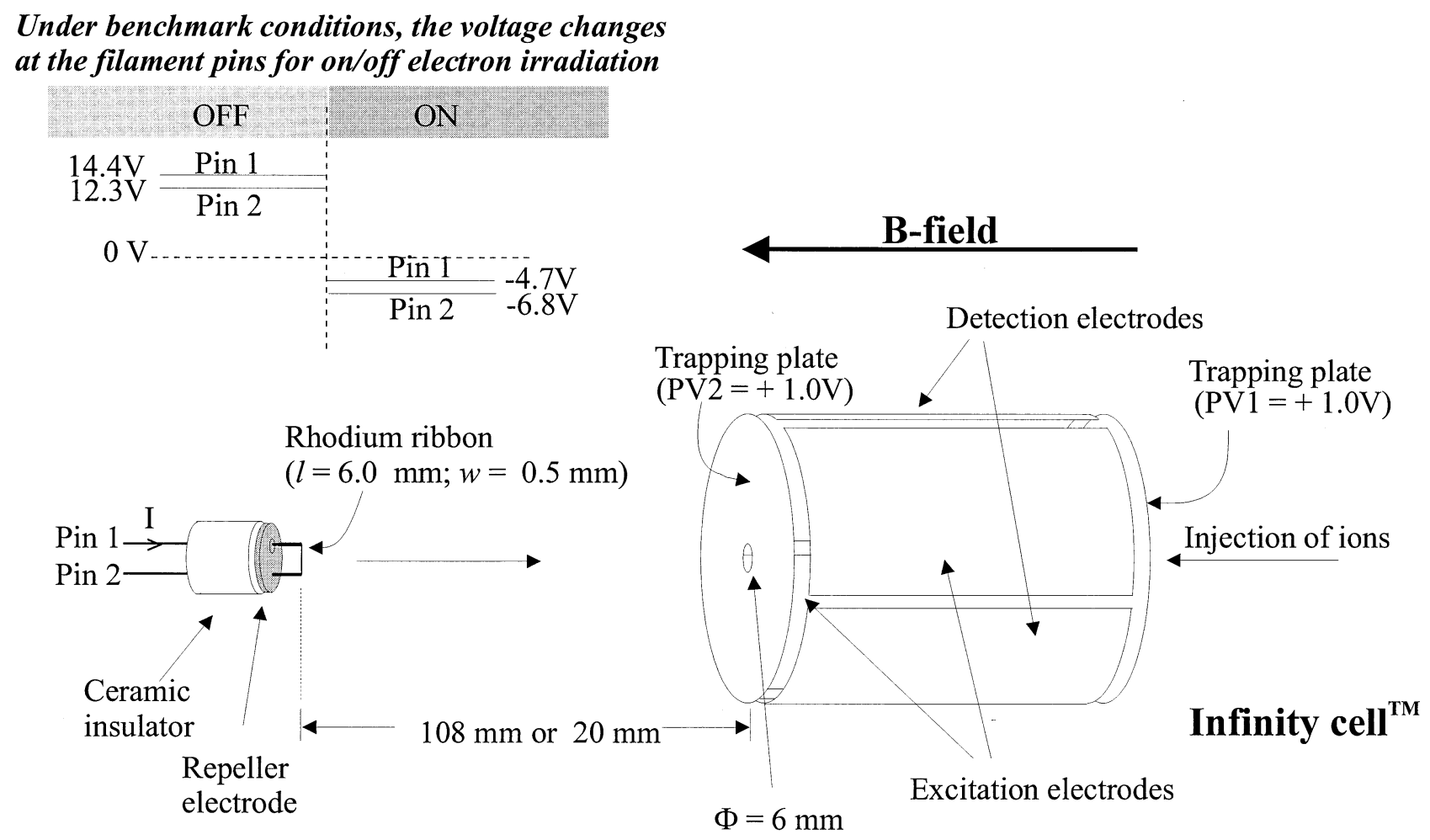

Figure 1. A schematic diagram of the electrically heated filament and the trapped ion cell.

tion method might be attributed to (1) the low intensity of the ECD fragment peaks, and (2) the long electron irradiation time of 3-10 s for obtaining sufficient intensities for ECD fragment-ions. The current ECD protocol remains tedious and time-consuming. The low dutycycle has imposed additional constraints on the implementation of the ECD technique for use in on-line liquid chromatography MS/MS analysis [17, 18]. To improve the duty-cycle, Zubarev and co-workers have replaced the heated filament with an indirectly heated dispenser cathode [19]. With presumably a larger electron emission area, this electron emission system was found to produce good quality ECD spectra of isolated precursor ions with only $10 \mathrm{~ms}$ electron irradiation.

To promote a wider utility of this powerful ion dissociation technique, we have used a commercially available Fourier-transform ion-cyclotron-resonance mass spectrometer (FTMS) system and conducted a thorough study of the impact of various experimental conditions on the efficiency of electron capture induced dissociation of peptide ions. Similar to many researchers, a resistive heated filament (for in-cell electron impact ionization experiments) was used to produce electrons for ECD experiments.

\section{Experimental}

\section{Sample Preparation}

All materials were obtained commercially and were used without further purification. Substance $P$, brady- kinin, luteinizing hormone releasing hormone (LHRH), bombesin, and carrisin were obtained from Sigma Chemical Co. (St. Louis, MO). Methanol (HPLC grade) was obtained from Labscan Ltd. (Bangkok, Thailand). All analyte solutions were prepared at concentrations of $25-50 \mathrm{pmol} / \mu \mathrm{L}$ in 1:1 methanol/water ( $\mathrm{vol} / \mathrm{vol}$ ), with the addition of $1 \%$ acetic acid.

\section{Instrumentation}

All experiments described in this paper were performed by using a 4.7 tesla FTMS system (APEX I, Bruker Instrument Inc., Boston, MA) equipped with an unshielded 4.7 tesla superconducting magnet and a standard commercially available electrospray ion source (Analytica, Branford, CT). Details of the instrumental arrangement have been reported previously [20]. Although previous reports [18, 21] using similar FTMS instrument have stressed the importance of using gated-trapping method [22] to confine the accumulated ion packet on the magnetic field axis for ECD experiments, the conventional sidekick ion accumulation method was used in the present study. Good quality ECD spectra could still be obtained. The FTMS instrument was equipped with a standard electrically heated filament source for electron emission as shown in Figure 1. With different filament supports, the filament position $\left(f_{p}\right)$ could be adjusted to either a distance of $108 \mathrm{~mm}$ or 20 $\mathrm{mm}$ from the rear end of the INFINITY cell [23] using a home-made adapter flange. The trapped ion cell was 
Table 1. A comparison of the literature experimental conditions and the benchmark conditions used in the present study for electron capture dissociation of multiply-charge protein/peptide ions

\begin{tabular}{lll}
\hline & \multicolumn{1}{c}{ Literature recommendation } & The benchmark conditions \\
\hline \hline Magnetic field strength & Range from 4.7 to 9.4 tesla & 4.7 tesla \\
Electron emission system & $\begin{array}{l}\text { Heated filament; and } \\
\text { Indirectly heated dispenser cathode }\end{array}$ & Heated filament \\
Filament current & Critical but vary from system to system & $3.0 \mathrm{~A}$ \\
Pulsing of Cooling gas & Optional (if use, then $\mathrm{Ar}>\mathrm{N}_{2}>\mathrm{Kr}$ ) & $\mathrm{Nil}$ \\
Electron irradiation & 3 to 20 seconds (for filament-type electron emission system) & $3 \mathrm{~s}$ \\
& 10 milliseconds ${ }^{\mathrm{a}}$ (for indirectly heated dispenser cathode) & $5.75 \mathrm{eV}$
\end{tabular}

${ }^{\mathrm{a}}$ For ECD of isolated precursor ions.

cylindrical with a dimension of $60 \mathrm{~mm}(\phi) \times 60 \mathrm{~mm}(l)$. Both trapping plates have annular holes of $6 \mathrm{~mm}(\phi)$. The filament was made of a Rhodium ribbon of width $\sim 0.5 \mathrm{~mm}$ and was spot-welded onto two metal posts (pin 1 and pin 2) with a separation of $\sim 6 \mathrm{~mm}$. The filament resistance was $0.5 \Omega$ under ambient conditions. Under typical conditions with no filament heating current and electron irradiation, equal potentials of +13.2 $\mathrm{V}$ were loaded onto the connection pin 1 and pin 2 . At a filament heating current of $3.0 \mathrm{~A}$ (see Table 1 for the benchmark conditions), the potentials at pin 1 and pin 2 were changed to $+14.4 \mathrm{~V}$ and $+12.3 \mathrm{~V}$, respectively. To emit electrons for ECD process(es) under benchmark conditions (i.e., $5.75 \mathrm{eV}$ electron energy), the potentials at pin 1 and pin 2 were ramped down to $-4.7 \mathrm{~V}$ and $-6.8 \mathrm{~V}$, respectively. A metallic plate was placed at the back of the filament and was electrically connected to the pin 2. Being at a lower potential (i.e., $-6.8 \mathrm{~V}$ ) than the average filament potential (i.e., $-5.75 \mathrm{eV}$ ), the metallic plate repelled the electrons towards the trapped ion cell. All ECD mass spectra were acquired in broadband mode using 128 Kbyte data set. The timedomain signals were zero-filled twice prior to Fourier transformation.

\section{Results and Discussion}

\section{Benchmark Conditions}

Table 1 summarizes common ECD experimental conditions and the so-called "benchmark conditions". The benchmark conditions were extracted from experimental parameters published in literatures by various research groups. Since an electrically heated filament was used to produce electrons in the present ECD experiments, only experimental parameters defining the electron energy and electron irradiation time have been adopted. Since suitable filament heating current depends on the filament materials and geometry, a benchmark heating current of 3.0 A was obtained from a series of preliminary experiments. Figure 2 shows typical electron capture dissociation mass spectra of molecule-ions of (Figure 2a) substance $P$, (Figure 2b) bombesin, and (Figure 2c) melittin obtained using benchmark conditions with filament position $\left(\mathrm{f}_{p}\right)$ of $108 \mathrm{~mm}$. The electron capture dissociation of the isolated doubly- protonated molecule ions of substance $\mathrm{P}$ shows abundant unreacted precursor ions with some low intensity ECD-induced fragment ions. Consistent with literature findings, all c-fragments (except $\mathrm{c}_{1}^{+}$and $\mathrm{c}_{3}^{+}$) can clearly be identified [25]. The lack of $\mathrm{c}_{1}^{+}$and $\mathrm{c}_{3}^{+}$ions can be explained by the cyclic structure of the proline which prohibits the corresponding backbone cleavage at the $\mathrm{N}$-terminal size of the proline residues [8]. Using similar experimental conditions, the ECD MS/MS mass spectrum of isolated doubly-protonated molecule-ions of bombesin shows only limited expected ECD-induced fragment ions (see Figure $2 b$ ). Only major fragment ions, i.e., $\mathrm{c}_{5}^{+}$to $\mathrm{c}_{8}^{+}$were observed. For a larger peptide, the ECD MS/MS mass spectrum of the isolated tetraprotonated melittin molecule-ions shows very limited number of ECD-induced fragments. The percentage of cleavage of inter-residue linkages was found to be $\sim 32 \%$.

Using benchmark conditions, our preliminary results are consistent with literature reports using similar commercial instrument $[17,18,21,24]$. The electron caption dissociation efficiency of multiply-charge peptide ions was not very high. Among various peptide/protein standards evaluated, only small peptides (MW $\sim 1000$ Da) show substantial ECD-induced fragmentation. For larger peptides such as bombesin and melittin, ECD of the isolated multiply-protonated precursor ions $[\mathrm{M}+$ $\mathrm{nH}]^{\mathrm{n}+}$ exhibit the reduced species, such as $[\mathrm{M}+$ $\mathrm{nH}]^{(\mathrm{n}-1)+\cdot}$ ions, with limited number of fragment ions. In contrast to some literature results, no ECD-induced fragment was observed for protein standards like bovine insulin or ubiquitin under the benchmark experimental conditions. Attempts to induce and/or increase the abundance of ECD-induced fragments by using higher flux of electrons (with higher filament current) result in complete quenching of the trapped ions, including both precursor and fragment ions.

\section{Effect of the Filament Position $\left(f_{p}\right)$ and Pulse Cooling Gas}

Figure 3 shows typical electron capture dissociation mass spectra of molecule-ions of (Figure $3 a$ ) substance $P$ and (Figure $3 b$ ) bombesin. In both cases, the top spectra were obtained after the filament position $\left(\mathrm{f}_{p}\right)$ was ad- 


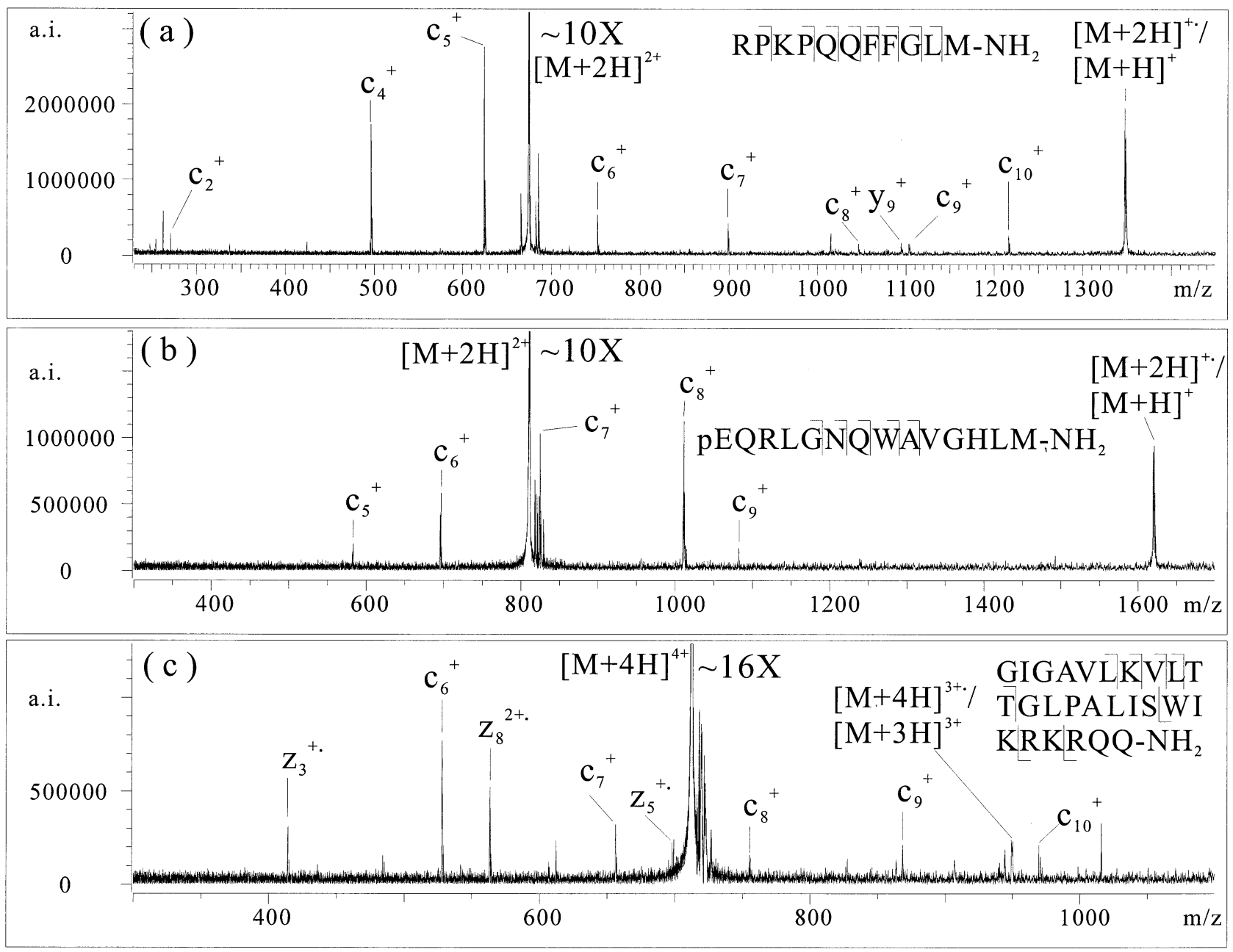

Figure 2. Typical electron capture dissociation mass spectra of isolated doubly-protonated ions of (a) substance $P,(\mathbf{b})$ bombesin, and (c) melittin obtained using benchmark conditions as shown in Table 1 .

justed to $\sim 20 \mathrm{~mm}$ away from the rear trapping plate of the trapped ion cell using the benchmark conditions, whereas the bottom spectra were obtained under the same conditions, i.e., benchmark conditions and $\mathrm{f}_{p}=20$ $\mathrm{mm}$, but with an additional pulse of argon gas during the electron irradiation event. During the acquisition of the bottom spectra, the pressure of the trapped ion cell was temporarily raised to $5 \times 10^{-7}$ torr using a $1150 \mu \mathrm{s}$ gas pulse. After the $3 \mathrm{~s}$ electron irradiation, an additional $5 \mathrm{~s}$ pumping delay was also included in the pulse program. The pressure of the trapped ion cell was reduced to less than $2 \times 10^{-8}$ torr prior to ion excitation and detection. Comparing the top spectra of Figure $3 a$ and $b$ with that of the Figure $2 a$ and $b$, moving the filament closer to the trapped ion cell showed some improvements on the quality of the ECD mass spectra. This might be a consequence of the higher transmission of electrons from the filament surface into the trapped ion cell. No observable change was, however, found in the fragmentation pattern.

Increasing the pressure of the trapped ion cell by purging with inert argon gas during the electron irra- diation was found to exert significant improvement in the quality of the ECD spectra for both substance $P$ and bombesin. For instance, all predicted ECD-induced sequence specific fragments of bombesin, i.e., $C_{3}^{+}-C_{13}^{+}$, could be clearly observed. Similar to earlier findings, nitrogen and krypton have found to give inferior performance compared to argon (data not shown) [25]. The exact function of the pulse gas was not known. It was suggested that argon gas might either reduce the kinetic energy of the injected electrons and hence improving the electron capture efficiency [25]; and/or reduce the kinetic energy of the ECD-induced fragment ions and hence improving the trapping efficiency of the fragment ions [25]. Nevertheless, it was clearly demonstrated that a better ECD spectrum could be obtained by using pnematically-assisted conditions.

\section{Effect of the Filament Heating Currents $\left(I_{f}\right)$}

The ECD efficiency of the doubly-protonated substance $P$ molecule-ions was investigated as a function of the filament heating current $\left(\mathrm{I}_{f}\right)$. The filament position $\left(\mathrm{f}_{p}\right)$ 


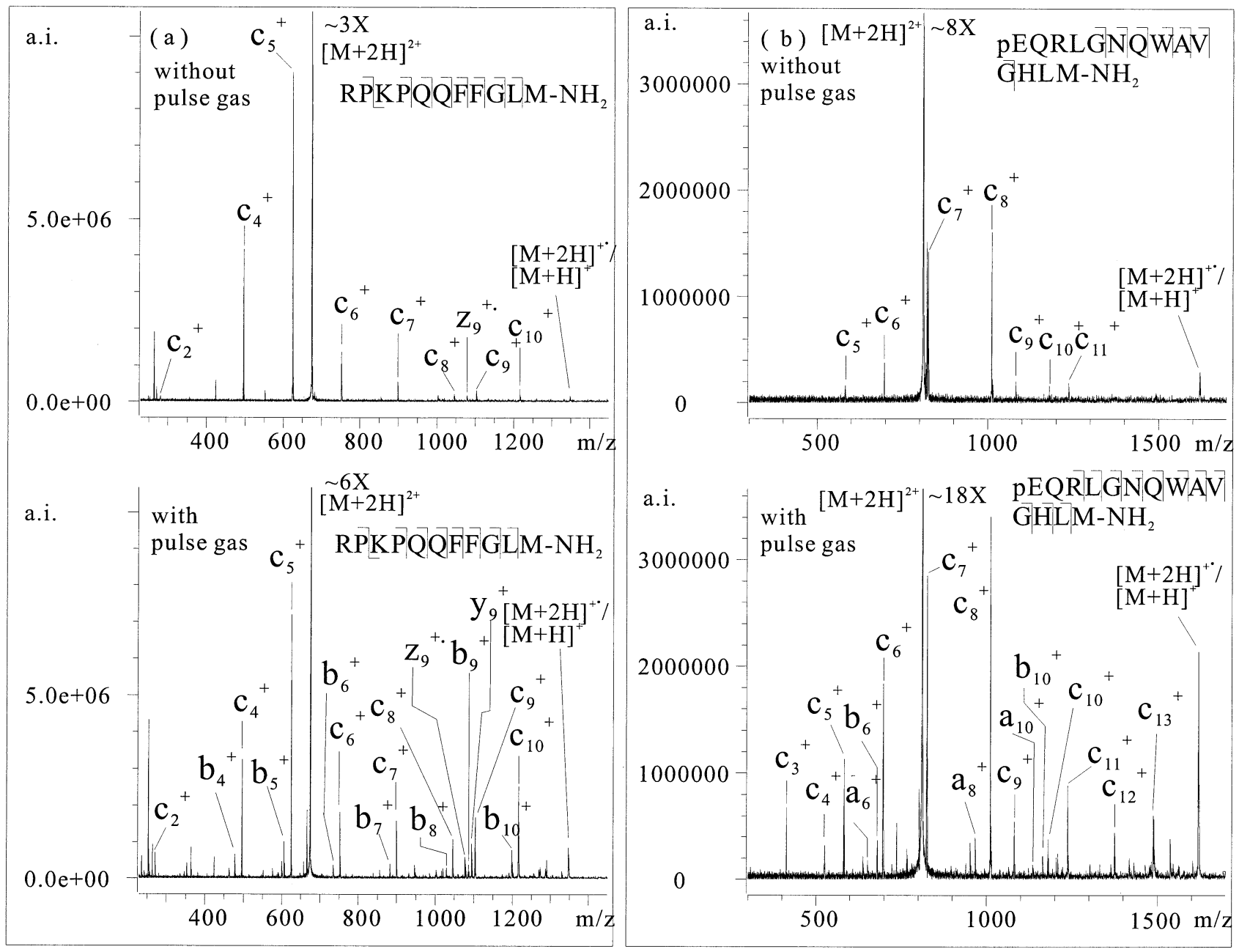

Figure 3. Electron capture dissociation mass spectra of isolated doubly-protonated ions of (a) substance $P$ and (b) bombesin obtained using a filament position of $20 \mathrm{~mm}$ with (bottom spectra) and without (top spectra) pulsing of argon cooling gas (1150 $\mu$ s gas pulse; pressure rise up to $5 \times 10^{-7}$ torr).

was fixed at $108 \mathrm{~mm}$. Figure 4 shows the intensity variation of the substance $P$ molecular ions $([\mathrm{M}+$ $2 \mathrm{H}]^{2+}$ ) and its ECD-induced fragment ions (i.e., $\mathrm{c}_{2}^{+}-\mathrm{c}_{10}^{+}$, except $\mathrm{c}_{3}^{+}$) as a function of the filament heating current. The same set of experiments was repeated three times to obtain statistical information. Data obtained from each replicate were normalized with respect to the sum of the ion intensities obtained across the range of filament heating current examined. The mean and standard deviation (i.e., the error bar) of the three normalized replicates was computed. Relative intensity was obtained by dividing the intensity of each peak with that of the highest intensity and was plotted against the filament heating current. The average filament bias voltage and electron irradiation time were fixed at $6.0 \mathrm{~V}$ and $3.0 \mathrm{~s}$, respectively, throughout the series of experiments.

From the series of plots in Figure 4, the signal intensities of the precursor ions and the ECD fragments are highly correlated across the range of filament current examined. At filament heating current $(<2.5 \mathrm{~A})$, the precursor ion intensity remained almost constant. None of expected ECD-induced fragment ions was formed at these low filament heating currents. This is consistent with a notion that emission of electrons from the filament was not substantial at low heating current $(<2.5 \mathrm{~A})$ under a moderately low electric field $(<300$ $\mathrm{Vm}^{-1}$ ). Increasing the filament heating current beyond 2.6 A led to a rapid drop in the intensity of the substance $P$ molecule-ions with a concomitant rise in the intensities of ECD-induced fragment ions. However, it was interesting to note that the precursor ion signal did not drop continuously as the filament heating current was increased. The signal intensity was found to level off at around 3.1 A. Further increase in the filament heating current did not have much impact on the residual precursor ion intensity. It was also intriguing to find that the impact of the filament heating current on the signal intensities of the ECD-induced fragments was somehow correlated with their sizes. There seems to be an optimum heating filament current for the formation of high-mass ECD fragments, i.e., 

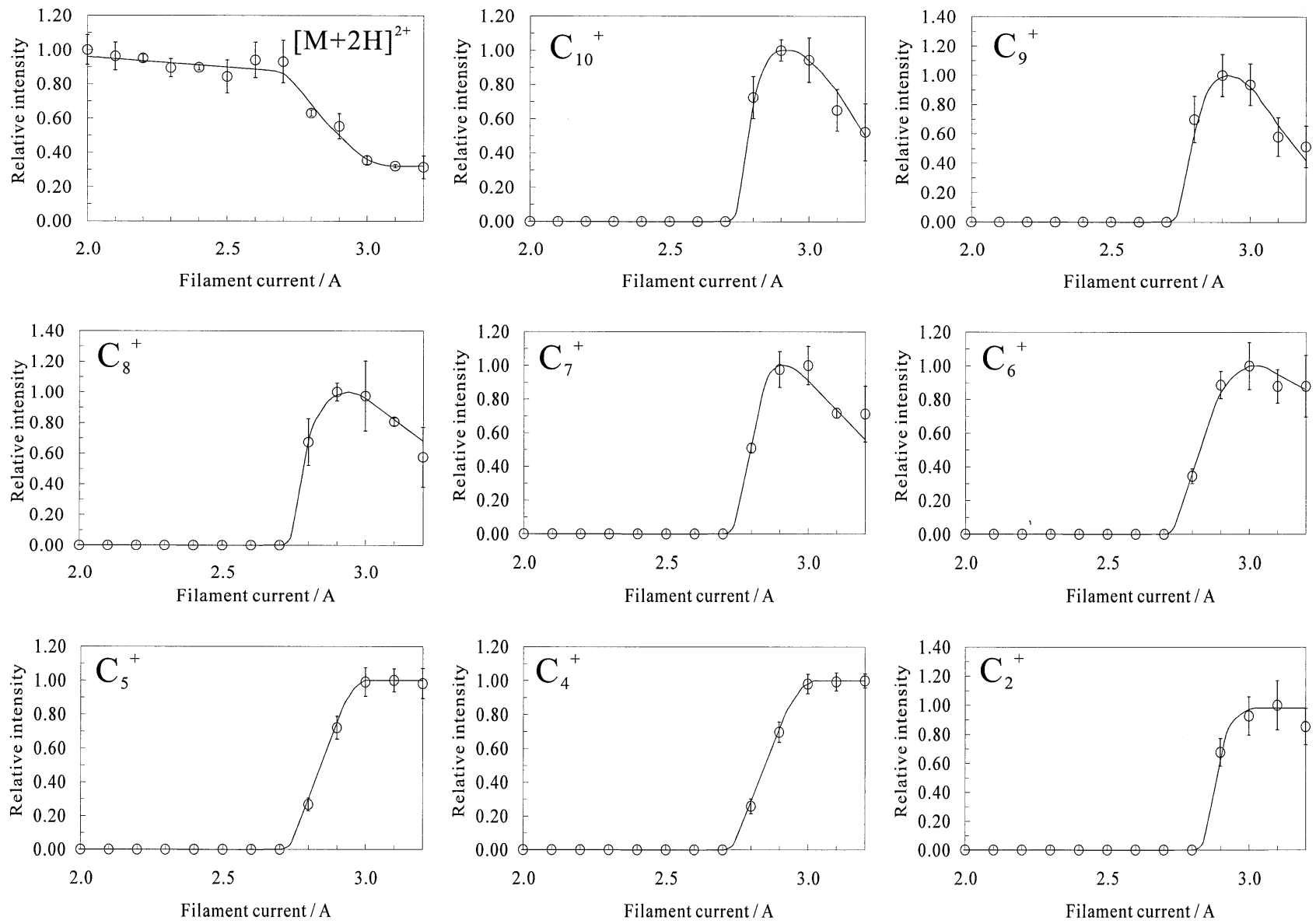

Figure 4. Effect of the filament heating current on the depletion of the isolated doubly-protonated ions of substance $P$ (i.e., the precursor ion) and the formation of the various electron capture induced fragment ions.

$\mathrm{c}_{6}^{+}-\mathrm{c}_{10}^{+}$ions, above which the intensities of these fragment ions decrease with increasing filament heating current. In contrast, the signal intensities of low-mass $\mathrm{ECD}$ fragments, i.e., $\mathrm{c}_{2}^{+}-\mathrm{c}_{5}^{+}$, increase with filament heating current to a maximum and level off at higher filament heating current. The drop of the precursor ion intensity with the concomitant rise in fragment ions intensities at $\mathrm{I}_{f}$ of 2.7-3.1 A is consistent with the increasing flux of electron emission from the filament into the trapped ion cell. The unexpected leveling of the precursor ion signal at $\mathrm{I}_{f}$ larger than $3.1 \mathrm{~A}$ could be explained on the basis of the cross section of the electron beam and the distribution of the precursor ions within the trapped ion cell. Because of the presence of a strong magnetic field (4.7 tesla), electrons are expected to transverse spirally along the magnetic field line with little displacement at $\mathrm{x}$ - and $\mathrm{y}$-directions (i.e., $\Delta \mathrm{x}=\Delta \mathrm{y} \approx 0$ ). Since the emitting surface of the filament is much smaller $(<6 \mathrm{~mm})$ than the dimension of the trapped ion cell $(\phi=60 \mathrm{~mm})$, the ion cloud within the cell is very likely to have a much large dimension at $\mathrm{x}$ - and $\mathrm{y}$-axis as compared with the cross section of the electron beam. However, only ions oscillating within and/or at the proximity of the electron beam would capture electrons.
It is also reasonable to postulate that only part of this overlapping ion population would capture electrons and would be reduced at low electron flux (i.e., $\mathrm{I}_{f} \sim 2.6$ ). Increasing the electron flux by increasing the $\mathrm{I}_{f}$ from 2.6 A. to $3.1 \mathrm{~A}$ would lead to a higher probability of reducing this overlapping ion population. Due presumably to the low capture cross section, those ions that are not oscillating within or at the proximity of the ion beam would not capture electrons even under high flux conditions. At $\mathrm{I}_{f}$ of $\sim 3.1 \mathrm{~A}$, nearly all precursor ions along the electron beam path would have captured electrons and undergone dissociation. Further increase in the electron flux by using higher $\mathrm{I}_{f}$ would not further reduce the precursor ion signal. The rise in the intensities of the reduced precursor ions and ECD-induced fragment ions (see Figure 4) at If of 2.6-3.1A is consistent with the above postulation. At $\mathrm{I}_{f}$ larger than 3.1A, high-mass fragment ions $\left(\mathrm{c}_{6}^{+}-\mathrm{c}_{10}^{+}\right)$and low-mass fragment ions $\left(\mathrm{c}_{2}^{+}-\mathrm{c}_{5}^{+}\right)$showed distinct behaviors. The intensities of high-mass fragment ions were found to drop with increasing $\mathrm{I}_{f}$; whereas the intensities of low-mass fragment ions showed no significant changes at higher $\mathrm{I}_{f}$. The drops in intensities of high-mass fragment ions were tentatively attributed to the neutralization of the 

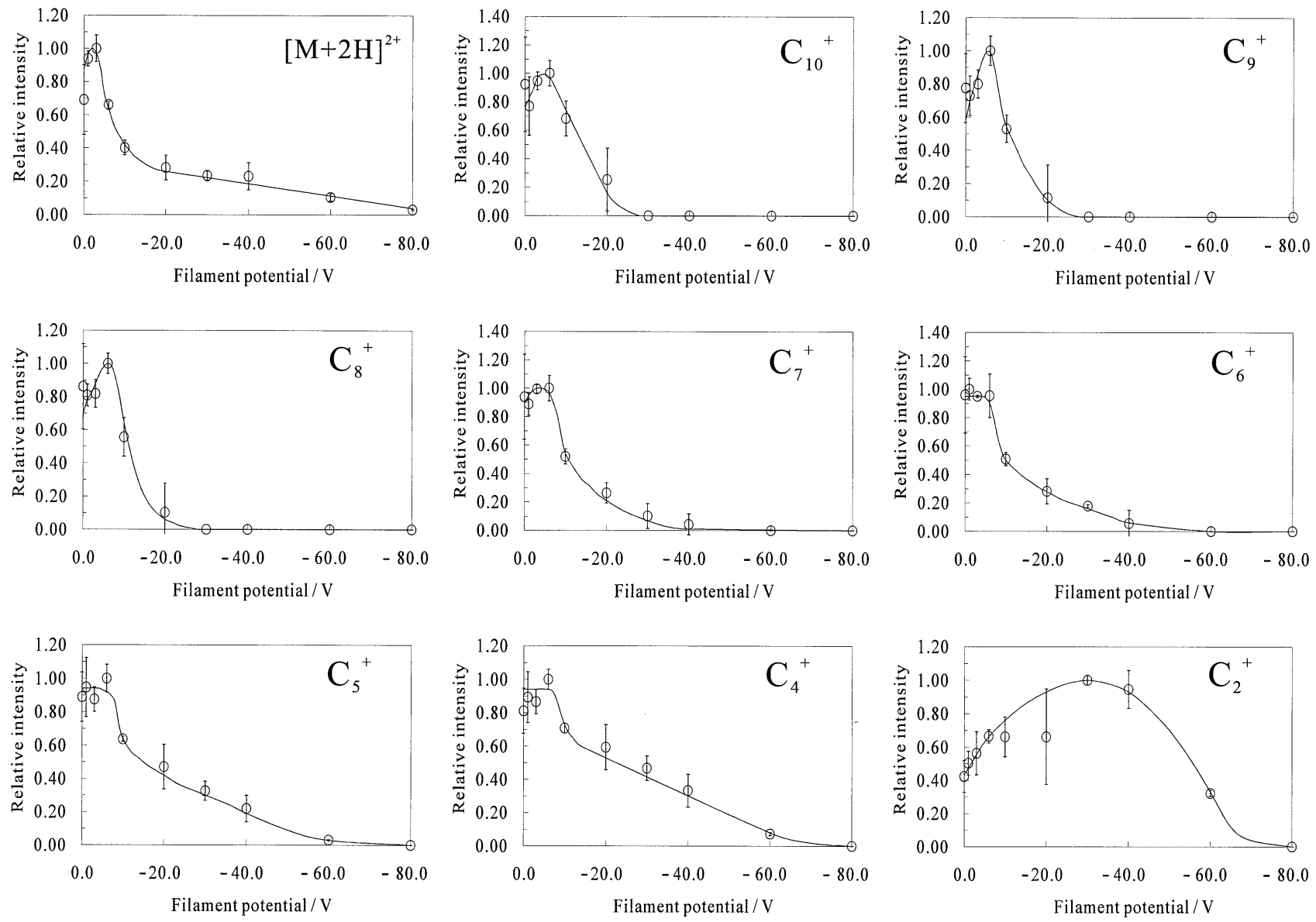

Figure 5. Effect of the electron energy on the depletion of the isolated doubly-protonated ions of substance $P$ (i.e., the precursor ion) and the formation of the various electron capture induced fragment ions.

reduced precursor ions and/or singly-charge fragment ions by capturing an additional electron. Due presumably to the smaller electron capture cross section, small fragment ions might have little affinity to capture additional electrons under the present experimental conditions.

\section{Effect of the Average Filament Bias Voltages $\left(V_{f}\right)$}

The ECD efficiency of doubly-protonated substance $P$ molecule-ions was also investigated as a function of the average filament bias voltages $\left(\mathrm{V}_{f}\right)$ (and hence the electron energy). The filament position $\left(f_{p}\right)$ was fixed at $20 \mathrm{~mm}$. The experiments were also repeated three times to obtain statistical information. Figure 5 shows the intensity variation of the substance $P$ molecular ions $\left([\mathrm{M}+2 \mathrm{H}]^{2+}\right)$ and the ECD-induced fragment ions (i.e., $\mathrm{c}_{2}^{+}-\mathrm{c}_{10}^{+}$, except $\left.\mathrm{c}_{3}^{+}\right)$as a function of the average filament bias voltages. The error bars were obtained from \pm standard deviation of the normalized replicates. The filament heating current and the electron irradiation time were maintained at $3.0 \mathrm{~A}$ and $3.0 \mathrm{~s}$, respectively, throughout the whole series of experiments. $V_{f}$ was computed by averaging the voltages applied onto the two filament pins. Because of the potential drop across the filament, the energy of the emitted electron is a variable depending on the site of electron emission, i.e., $\pm 1.05 \mathrm{~V}$, and its thermal energy.

From the series of plots in Figure 5, several interesting features relating to the ECD process(es) were revealed. The signal intensities of the precursor ions and ECD-induced fragment ions were influenced by $V_{f}$ at different extents. At zero $\mathrm{V}_{f}$, the intensity of the substance $P$ molecule-ions was reduced approximately by $50 \%$ in comparison with the intensity of the same ions on which no electron irradiation was performed. Concurrently, abundant ECD-induced fragments were observed. For the precursor ions and the high-mass fragment $\left(\mathrm{c}_{10}^{+}-\mathrm{c}_{7}^{+}\right)$ions, there was a sharp rise in signal intensities when $\mathrm{V}_{f}$ was decreased from $0 \mathrm{~V}$ to approximately $-6 \mathrm{~V}$. No such rise was observed for low-mass fragment ions $\left(\mathrm{c}_{4}^{+}-\mathrm{c}_{6}^{+}\right)$. Decreasing $\mathrm{V}_{f}$ beyond $-6 \mathrm{~V}$, sharp decreases in the ion signals was found for almost all ions (except $\mathrm{c}_{2}^{+}$). For the precursor ions, the rate of reduction of the signal intensity was much lower at $\mathrm{V}_{f}$ lower than $-20 \mathrm{~V}$. Similar trends were observed for ECD-induced fragment ions. However, high-mass fragment ions were diminished to such an extent that they 
were no longer visible in the typical ECD spectra at $\mathrm{V}_{f}$ lower than $-30 \mathrm{~V}$. Detectable signals could be observed for smaller fragment ions $\left(\mathrm{c}_{6}^{+}-\mathrm{c}_{4}^{+}\right)$at $\mathrm{V}_{f}$ as large as -60 $\mathrm{V}$. The smallest ECD-induced fragment, $\mathrm{c}_{2}^{+}$, behaved rather differently. Its intensity was gradually raised by decreasing the $\mathrm{V}_{f}$ from zero to $\sim-30 \mathrm{~V}$ and was diminished slowly to zero at $-80 \mathrm{~V}$.

The large drop in the precursor ion intensity at zero $\mathrm{V}_{f}$ is consistent with earlier findings that the electron capture cross-section $(\sigma)$ is optimized at near-zero translational energy difference between the trapped ions $(<1.0 \mathrm{eV})$ and irradiated electrons $(<0.9 \mathrm{eV})$ [25]. However, it is important to note that the signal intensities for the precursor ions and ECD-induced fragment ions were not very stable. This was attributed to the relatively inefficient emission of electrons from the filament surface and/or low transmission efficiency of electrons through the aperture of the rear plate of the trapped ion cell.

The initial rise in the intensity of the precursor ions and the high-mass ECD-induced fragment ions at low $\mathrm{V}_{f}(0 \mathrm{~V}$ to $-6 \mathrm{~V})$ was intriguing. The rise in the intensity of the precursor ion implies a reduction in the electron capture efficient. This is consistent with the theory that increasing energy mismatch between the trapped ions and electron energy reduces the electron capture cross section. However, reduction in the electron capture efficient should in principle reduce the intensity of ECD-induced fragment ions rather than increase the fragment ions formation. A possible explanation for this unexpected result involves the consideration of the energy and spatial distribution of the precursor ions within the trapped-ion cell, and the possible neutralization of the reduced precursor ions and singly-charge fragment ions by capturing additional electrons. In line with the argument made in a previous section, we made a postulation that the dimension of the ion cloud within the trapped ion cell is larger than the cross section of the electron beam along the $\mathrm{x}$ - and $\mathrm{y}$-axis. At near zero $\mathrm{V}_{f}$, the near zero energy electrons have high affinity to be captured by the spatial overlapping ion cloud. After initial capturing of the electrons, the reduced precursor ion and its fragment ions could capture second electrons to form neutral species. The fragment ions intensities observed at near zero $\mathrm{V}_{f}$ (see Figure 5) were already diminished because of the neutralization process. Decreasing the $\mathrm{V}_{f}$ from $0 \mathrm{~V}$ to $-6 \mathrm{~V}$ reduced the capture cross-section for the trapped ions and the precursor ion intensity was increased. The corresponding increase in the high-mass ECD-induced fragments was actually a consequence of the lower possibility of fragment ions to capture additional electron, i.e., lower possibility of neutralization loss. The absence of similar increase for low-mass ECD-induced fragments might be attributed to the small sizes of these ions, i.e., low capture cross-section. The falling of the intensity of the precursor ions and the ECD-induced fragment ions at moderate to high $\mathrm{V}_{f}(-6 \mathrm{~V}$ to $-80 \mathrm{~V})$ was another interesting observation. In theory, decreasing $\mathrm{V}_{f}$ (more negative) has two major impacts. It increases (1) the average kinetic energy of the electrons to high values and hence reducing the electron capture cross-section, and (2) the electron flux by extracting more electrons per unit time from the heated filament. Assuming that the increased electron flux has no impact on the trapping efficiency of the trapped ion cell, the factor relating to the increase in electron flux might outweigh the one for the reduction of electron capture cross-section. This would account for the decreasing trend of precursor ion intensity at $\mathrm{V}_{f}$ lower than $-6 \mathrm{~V}$. Considered solely on the dissociative recombination pathway, a reduction in the precursor ion intensity should accompany an increase in the intensity of ECD-induced fragment ions. However, if one takes into the consideration the possibility of neutralization loss, the signal intensity of ECD-induced fragment ions might also be reduced by lowering the $\mathrm{V}_{f}$ (more negative). Since the neutralization loss involves subsequent capture of another electron by the reduced precursor and fragment ions, the rate of neutralization loss might differ from ions to ions depending on their electron capture cross-sections (or physical size of the ions). This is in fact observed in Figure 5 that low-mass ECD-induced fragment ions were reduced at a much slower rate than the high-mass ions. From the analytical point of view, the present results illustrate that good ECD-mass spectra of peptide ions could be registered at a wide range of average filament bias voltages (and hence the electron energies) even though the electron capture cross section $(\sigma)$ is very sensitive to the energy of the electrons.

It is perhaps worthwhile to note that apart from the consideration made in the signal intensities of various ions, the nature of fragment ions observed in our ECD spectra using different $\mathrm{V}_{f}$ were almost identical. Fragment ions signifying hot electron capture dissociation (HECD) [26] and electron excitation dissociation (EED) [27] of the precursor ions were not observed. The apparent lack of these dissociation processes might be attributed to the comparatively low-electron flux density of the filament-based electron gun compared with the indirectly-heated cathode dispenser [19].

\section{Effect of the Electron Irradiation Time}

The ECD efficiency of doubly-protonated substance $P$ molecule-ions was investigated as a function of the electron irradiation time $\left(\mathrm{t}_{e}\right)$. The filament position $\left(\mathrm{f}_{p}\right)$ was fixed at $20 \mathrm{~mm}$. The experiments were also repeated three times. Figure 6 shows the intensity variation of the substance $P$ molecular ions $\left([\mathrm{M}+2 \mathrm{H}]^{2+}\right)$ and its ECD-induced fragment ions (i.e., $\mathrm{c}_{2}^{+}-\mathrm{c}_{10}^{+}$, except $\mathrm{c}_{3}^{+}$) as a logarithmic function of the electron irradiation time, i.e., $\ln \left(t_{e}\right)$. The error bars were obtained from \pm standard deviation of the normalized replicates. In these experiments, the energy and the flux of electrons were held almost constant by using a fixed filament heating current (3.0 A) and filament bias voltages (6.0 $\mathrm{V})$. Under these conditions, the electron-capture cross- 

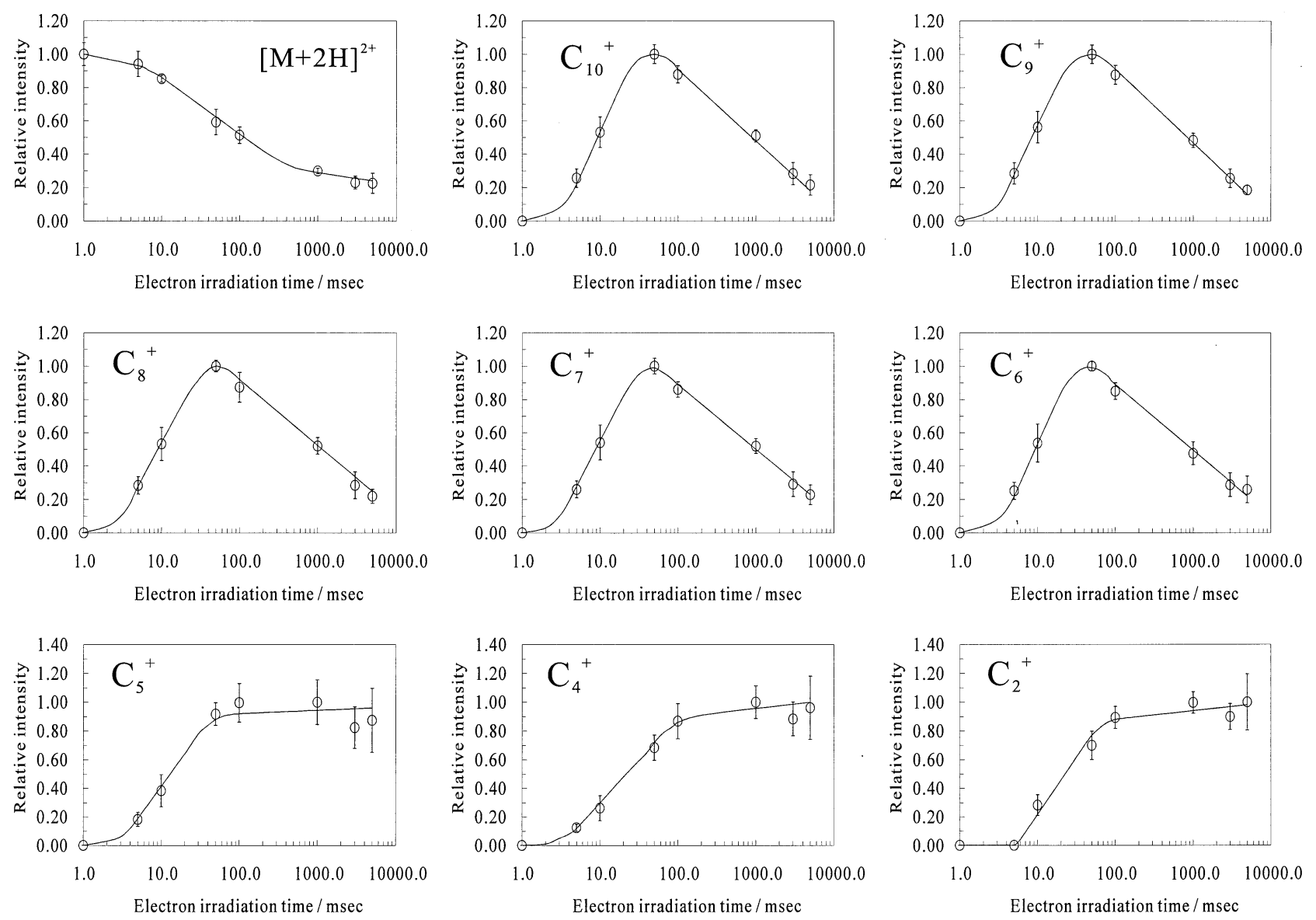

Figure 6. Effect of the electron irradiation time on the depletion of the isolated doubly-protonated ions of substance $P$ (i.e., the precursor ion) and the formation of the various electron capture induced fragment ions.

sections of the precursor ions and fragment ions should therefore be constants. The possibility of capturing electron by trapped ions should increase linearly with the electron irradiation time $\left(\mathrm{t}_{e}\right)$.

From the series of plots in Figure 6, it was interesting to note that the signal intensities of the precursor ions and ECD-induced fragment ions were strongly influenced by $\mathrm{t}_{e}$ using the electrically heated filament as electron emission source. At very short electron irradiation time $\left(\mathrm{t}_{e} \leq 1 \mathrm{~ms}\right)$, the intensity of the substance $\mathrm{P}$ molecule-ions showed insignificant changes. No detectable ECD-induced fragments were observed. At $t_{e}$ of 5 $\mathrm{ms}$, a small drop of the precursor ion signals was observed with the production of small but significant ECD-induced fragments. Increasing $\mathrm{t}_{e}$ from $5 \mathrm{~ms}$ up to 1 second resulted in an exponential decrease in the precursor ion intensity. Further increase in the $t_{e}$ beyond 1 second had little influence on the residual precursor ion intensity. Again, there seems to be a size dependence effect on the impact of $t_{e}$ on the intensities of the ECD-induced fragment ions. For the large fragment ions, i.e., $\mathrm{c}_{6}^{+}$to $\mathrm{c}_{10}^{+}$, their intensities were increased exponentially with $\mathrm{t}_{e}$ to a maximum at around $50 \mathrm{~ms}$. Above $50 \mathrm{~ms}$ of electron irradiation, their intensities dropped exponentially with $t_{e}$. For the small fragment ions, i.e., $c_{2}^{+}$to $c_{5}^{+}$, the signal intensities were also increased exponentially with $t_{e}$ up to around $50 \mathrm{~ms}$. Instead of reducing the fragment ion intensities, extending the electron irradiation time beyond $50 \mathrm{~ms}$ had no significant impact on the intensities of these small fragment ions. These interesting findings could again be explained on the basis of the distribution of the precursor ions with the trapped ion cell and the possible neutralization of the reduced precursor ions and singlycharge fragment ions by capturing additional electrons. Nevertheless, our experimental results indicate that good quality ECD spectra of peptides can be obtained by using filament-based electron gun with electron irradiation time as short as $10-50 \mathrm{~ms}$.

\section{Optimized Conditions}

Figure 7 show ECD MS/MS mass spectra of (Figure 7a) bombesin and (Figure $7 \mathrm{~b}$ ) carrasin using optimized experimental parameters with (bottom spectra) and without (top spectra) pulse cooling gas. With optimized experimental parameters; i.e., $\mathrm{V}_{f}=5.75 \mathrm{~V}, \mathrm{I}_{f}=3.0 \mathrm{~A}$, and $\mathrm{T}_{e}=50 \mathrm{~ms}$, ECD spectrum of bombesin shows 11 sequence specific fragment ions generated from cleavages of 9 out of 13 inter-residue linkages within the 


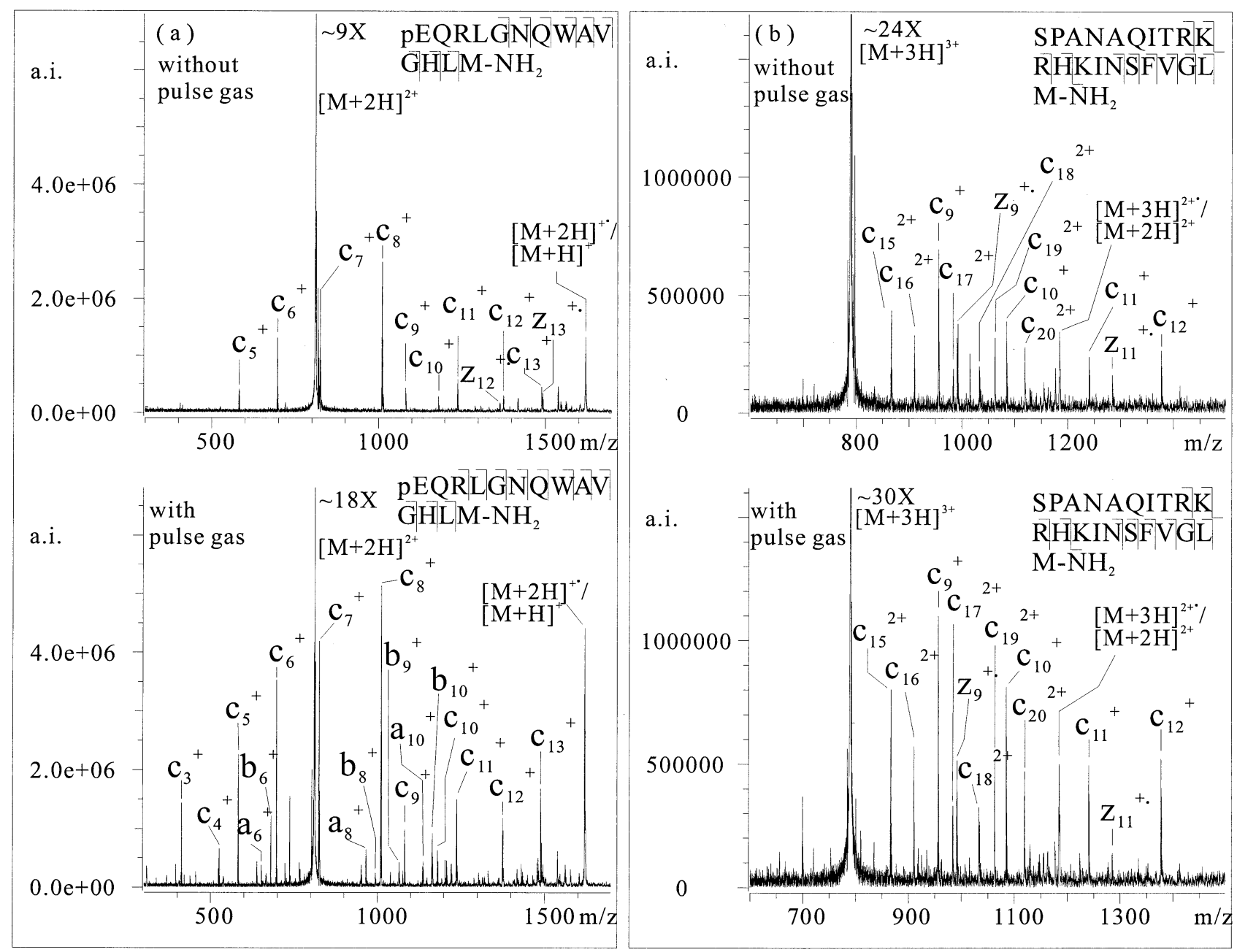

Figure 7. Electron capture dissociation mass spectra of isolated doubly-protonated ions of (a) bombesin and (b) carassin. Each peptide ion was analyzed under benchmark ECD conditions (1) with and (2) without pulsing of argon cooling gas (1150 $\mu$ s gas pulse; pressure rise up to $5 \times 10^{-7}$ torr). Electron irradiation time was reduced to $50 \mathrm{~ms}$.

molecules. Addition of the pulse of argon gas during the electron irradiation time doubled the number of sequence specific fragment ions including the formation of some aand $b$-ions. The percentage of cleavage of inter-residue linkages increased from 69 up to $85 \%$. Using this experimental arrangement, the ECD efficiency for larger peptide ions was, however, not particularly good. ECD spectrum of carrasin using optimized experimental conditions yields only 12 sequence specific fragment ions generated from cleavages of only 10 out of 20 inter-residue linkages within the molecules. The addition of pulse argon gas has some improvement on the overall ion signal intensities due presumably to the better trapping of the precursor and fragment ions. However, no noticeable improvement was found in terms of the number of sequence specific fragment ions or sequence coverage.

\section{Conclusions}

Through a systematic investigation of experimental parameters, the performance of a commercially avail- able Fourier-transform mass spectrometer for ECD experiments has been greatly improved. Quality ECD spectra of peptides with electron irradiation time as short as $50 \mathrm{~ms}$ without using the time consuming gas cooling procedure could be obtained. The relatively high performance of our heated filament electron gun for ECD of peptides (MW $<3000 \mathrm{Da}$ ) is not clearly understood. It is believed that the exact geometry of the heated filament might be an important parameter for efficient production and transmission of electrons into the trapped ion cell. Further work in characterizing the importance of the electron gun geometry on the efficiency of ECD is now in progress and will be published later.

\section{Acknowledgments}

The work described in this paper was supported by a grant (reference no. CUHK 4274/00P) from the Research Grants Council of the Hong Kong Special Administrative Region, China. 


\section{References}

1. Hayes, R. N.; Gross, M. L. Collision-Induced Dissociation. Methods Enzymol. 1990, 193, 237-263.

2. Little, D. P.; Speir, J. P.; Senko, M. W.; O'Connor, P. B.; McLafferty, F. W. Infrared Multiphoton Dissociation of Large Multiply Charged Ions for Biomolecule Sequencing. Anal. Chem. 1994, 66, 2809-2815.

3. Price, W. D.; Schnier, P. D.; Williams, E. R. Tandem Mass Spectrometry of Large Biomolecule Ions by Blackbody Infrared Radiative Dissociation. Anal. Chem. 1996, 68, 859-866.

4. Tsaprailis, G.; Nair, H.; Somogyi, A.; Wysocki, V. H.; Zhong, W.; Futrell, J. H.; Summerfield, S. G.; Gaskell, S. J. Influence of Secondary Structure on the Fragmentation of Protonated Peptides. J. Am. Chem. Soc. 1999, 121, 5142-5154.

5. Williams, E. R.; Furlong, J. J. P.; McLafferty, F. W. Efficiency of Collisionally-Activated Dissociation and 193-nm Photodissociation of Peptide Ions in Fourier-Transform Mass Spectrometry. J. Am. Soc. Mass Spectrom. 1990, 1, 288-294.

6. Roepstorff, P.; Fohlman, J. Proposal for a Common Nomenclature for Sequence Ions in Mass Spectra of Peptides. Biomed. Mass Spectrom. 1984, 11, 601.

7. (a)Vekey, K. Multiply Charged Ions. Mass Spectrom. Rev. 1995, 14, 195-225.(b)Yu, W.; Vath, J. E.; Huberty, M. C.; Martin, S. A. Identification of the Facile Gas-Phase Cleavage of the AspProd and Asp-xxx Peptide Bonds in Matrix-Assisted Laser Desorption Time-of-Flight Mass Spectrometry. Anal. Chem. 1993, 65, 3015-3023.

8. Zubarev, R. A.; Kelleher, N. L.; McLafferty, F. W. Electron Capture Dissociation of Multiply-Charged Protein Cations. A Nonergodic Process. J. Am. Chem. Soc. 1998, 120, 3265-3266.

9. Shi, S. D.-H.; Hemling, M. E.; Carr, S. A.; Horn, D. M.; Lindh, I.; McLafferty, F. W. Phosphopeptide/Phosphoprotein Mapping by Electron Capture Dissociation Mass Spectrometry. Anal. Chem. 2001, 73, 19-22.

10. Horn, D. M.; Ge, Y.; McLafferty, F. W. Activated Ion Electron Capture Dissociation for Mass Spectral Sequencing of Larger (42 kDa) Proteins. Anal. Chem. 2000, 72, 4778-4784.

11. Kelleher, N. L.; Zubarev, R. A.; Bush, K.; Furie, B.; Furie, B. C.; McLafferty, F. W.; Walsh, C. T. Localization of Labile Posttranslational Modifications by Electron Capture Dissociation: The Case of $\gamma$-Carboxyglutamic Acid. Anal. Chem. 1999, 71, $4250-4253$.

12. Mirgorodskaya, E.; Roepstoff, P.; Zubarev, R. A. Localization of O-Glycosylation Sites in Peptides by Electron Capture Dissociation in a Fourier Transform Mass Spectrometer. Anal. Chem. 1999, 71, 4431-4436.

13. Zubarev, R. A.; Kruger, N. A.; Fridrikson, E. K.; Lewis, M. A.; Horn, D. M.; Carpenter, B. K.; McLafferty, F. W. Electron Capture Dissociation of Gaseous Multiply-Charged Proteins is Favored to Disulfide Bonds and Other Sites of High Hydrogen Atom Affinity. J. Am. Chem. Soc. 1999, 121, 2857-2862.

14. Stensballe, A.; Jensen, O. N.; Olsen, J. V.; Haselmann, K. F.; Zubarev, R. A. Electron Capture Dissociation of Singly and Multiply Phosphorylated Peptides. Rapid Commun. Mass Spectrom. 2000, 14, 1793-1800.

15. Sze, S. K.; Ge, Y.; Oh, H.; McLafferty, F. W. Top-Down Mass Spectrometry of 29-kDa Protein for Characterization of Any
Posttranslational Modification to Within One residue. PNAS 2002, 99, 1774-1779.

16. Horn, D. M.; Zubarev, R. A.; McLafferty, F. W. Automated de Novo Sequencing of Proteins by Tandem High-Resolution Mass Spectrometry. PNAS 2000, 97, 10313-10317.

17. Palmblad, M.; Tsybin, Y. O.; Ramström, M.; Bergquist, J.; Håkansson, P. Liquid Chromtography and Electron Capture Dissociation in Fourier Transform Ion Cyclotron Resonance Mass Spectrometry. Rapid Commun. Mass Spectrom. 2002, 16, 988-992.

18. Davidson, W.; Frego, L. Micro-High-Performance Liquid Chromatography/Fourier Transform Mass Spectrometry with Electron-Capture Dissociation for the Analysis of Protein Enzymatic Digests. Rapid Commun. Mass Spectrom. 2002, 16, 993-998.

19. Tsybin, Y. O.; Håkansson, P.; Budnik, B. A.; Haselmann, K. F.; Kjeldsen, F.; Gorshkov, M.; Zubarev, R. A. Improved LowEnergy Electron Injection Systems for High Rate Electron Capture Dissociation in Fourier Transform Ion Cyclotron Resonance Mass Spectrometry. Rapid Commun. Mass Spectrom. 2001, 5, 1849-1854.

20. Lau, R. L. C.; Jiang, J.; Ng, D. K. P.; Chan, T.-W. D. Fourier Transform Ion Cyclotron Resonance Studies of Lanthanide(III) Porphyrin-Phthalocyanine Heteroleptic Sandwich Complexes by Using Electrospray Ionization. J. Am. Soc. Mass Spectrom. 1997, 8, 161-169.

21. Polfer, N. C.; Haselmann, K. F.; Zubarev, R. A.; LangridgeSmith, P. R. R. Electron Capture Dissociation of Polypeptides Using a 3 tesla Fourier Transform Ion Cyclotron Resonance Mass Spectrometer. Rapid Commun. Mass Spectrom. 2002, 16, 936-943.

22. Castro, J. A.; Koster, C.; Wilkins, C. Matrix-Assisted Laser Desorption/Ionization of High-Mass Molecules by Fouriertransform Mass Spectrometry. Rapid Commun. Mass Spectrom. 1992, 6, 239-241.

23. Caravatti, P.; Allemann, M. The Infinity Cell: A New TrappedIon Cell with Radiofrequency Covered Trapping Electrodes for Fourier Transform Ion Cyclotron Resonance Mass Spectrometry. Org. Mass Spectrom. 1991, 26, 514-518.

24. Axelsson, J.; Palmblad, M.; Håkansson, K.; Håkansson, P. Electron Capture Dissociation of Substance P Using a Commercially Available Fourier Transform Ion Cyclotron Resonance Mass Spectrometer. Rapid Commun. Mass Spectrom. 1999, 13, 474-477.

25. Zubarev, R. A.; Horn, D. M.; Fridriksson, E. K.; Kelleher, N. L.; Krugger, N. A.; Lewis, M. A.; Carpenter, B. K.; McLafferty, F. W. Electron Capture Dissociation for Structural Characterization of Multiply Charged Protein Cations. Anal. Chem. 2000, $72,563-573$.

26. Kjeldsen, F.; Haselmann, K. F.; Budnik, B. A.; Jensen, F.; Zubarev, R. A. Dissociative Capture of Hot (3-13 eV) Electrons by Polypeptide Polycations: An Efficient Process Accompanied by Secondary Fragmentation. Chem. Phys. Lett. 2002, 356, 201-206.

27. Nielsen, M. L.; Budnik, B. A.; Haselmann, K. F.; Olsen, J. V.; Zubarev, R. A. Intramolecular Hydrogen Atom Transfer in Hydrogen-Deficient Polypeptide Radical Cations. Chem. Phys. Lett. 2000, 330, 558-562. 